\title{
The Relations Between Classic And Geometric Probability and Scale-Free Feature In Social Networks
}

\author{
Fei $\mathrm{MA}^{1}$, Jing $\mathrm{SU}^{1}$ and Bing $\mathrm{YAO}^{1,{ }^{*}}$ \\ ${ }^{1}$ College of Mathematics and Statistics, Northwest Normal University, \\ Lanzhou, 730070, China \\ *Corresponding author: Email: yybb918@163.com.
}

Keywords: Probability, Scale-free, Power-law, Models, Ddifferential equation.

\begin{abstract}
Probability and statistics have been proved to be strong and useful tools for recovering the topological properties in complex systems and networks all over the world. We recall the process of studying complex systems in the past decades and propose general evolution equation about the dynamical function of network. Relations between classic and geometric probability and scale-free feature have been discussed, and we build up a class new network models in which the classic and geometric probability are coexisting.
\end{abstract}

\section{Introduction}

As known, in the past two decades studies of complex systems and networks have taken much attentions in wide variety of fields, from social science to biology to computer science. For example, the World-Wide-Web(WWW) [2], the internet, food webs [4], the collaboration networks [3], the web of sexual contacts [5], protein interaction networks [6], metabolic networks [7, 21], the scientific literature webs and so on. Indeed, it is a long time to research complex systems. Almost all these studies are based on the graphs which are a collections of vertices with edges connecting pairs of them $[9,10,13]$. The vertex and edges is stand for basic element and the relations of basic elements, respectively. A social-network is a collection of people, each of whom is communicating with some subsets of the others. The process of studying complex systems can be divided into three parts. Staring with the lightly influential work of Erdös and Rényi [15] came up with the stochastic network model, which is built at random, to describe complex network, called ER-model. As a new researching tool to complex networks, random graph theory has long-term growth into one the mainstays of computer science and modern discrete mathematics. At the end of the last century (in 1998s), Watts and Strogatz [16,18] put forward the small-world model (WS-model). Soon, based on the investigating on the topological structure of WWW, Barabási and Albert [17] presented firstly the model of scale-free network in their article published (in 1999s). The degree distribution of their model, hereafter named BA-model, satisfies the power-law formula: $P(k)=k^{-\gamma}, 2<k<3$. Dorogovtsev et.al. [8] gave $P_{\text {cum }}(k)=\sum_{k^{\prime} \geq k} N\left(k^{\prime}, t\right) / n_{v}(t) \approx k^{1-\gamma}$, called cumulative degree distribution, where $N\left(k^{\prime}, t\right)$ and $n_{v}(t)$ stands for the number of vertices with the degree $k^{\prime}$ and the order of network model $N(t)$ at time step $t$, respectively. Recently, a series of researching results [19, 22, $23,24,25]$ have shown that lots of complex network systems in society and nature have the scale-free property. 
The fact is universal to see the probability and statistics have proved to be strong and useful tools for recovering the topological properties in complex systems and networks which are not random but also deterministic $[11,12,14]$. The stochastic properties of hundreds of networks models are obtained by classic probability. This paper is organized as follows. In Sec 2, we propose general dynamical evolution equation about networks for depicting inner rules. In Sec 3, we discuss the relations between probability and scale-free feature emerging in the current networks models and then build up a class new network models in which the classic and geometric probability are coexisting. In Section 4, we make a brief summary and raise some meaningful and undetected problems to be taken over in the future.

\section{A Dynamical Partial Differential Equation}

We make some descriptions and states for the dynamical evolution process which contains vertices entered and removed, edges generated and removed and much interference from the outside at each time step. As time goes on, the scope of dynamical complex network become more and more complicate. The number vertices and edges increase rapidly and its spacial structure will become more complex. Nothing will always grow unceasingly. After undergoing an extended period, a network itself will tend to a stable or decay. Combining the real background meanings and theoretical analysis, we will make a simple depiction about the dynamical evolution of complex systems by introducing the following 5 characteristic functions [26,27].

(a) Adding vertex function $f^{*}(t) \cdot f^{*}(t)=f\left(a p_{1}(t) m, t, k_{i}(t), \sum \Pi_{1 j} k_{i}\right)$ indicates adding new vertices into the network $N(t-1)$ at time step $t$. A new vertex $j_{a}$ will devote $p_{1}(t) m\left(0<p_{1}(t)<1\right)$ edges to $N(t-1)$ by connecting with some old vertices. The probability which the degree $k_{i}(t)$ vertex obtains new links obeys the preferential attachment probability $\Pi_{1 j} k_{i}$, and $\sum \Pi_{1 j} k_{i}$ stands for all kinds of probability co-existing (the most important is preferential attachment probability).

(b) Removing vertex function $g^{*}(t)$. After the network goes through the fastly increasing process, $g^{*}(t)=g\left(p_{2}(t) b, t, k_{i}(t), \sum \Pi_{2 j} k_{i}\right)$ shows there are $p_{2}(t) b\left(0<p_{2}(t)<1\right)$ old vertices removed from $N(t-1)$ at time step $t$. An old vertex $j_{b}$ will be removed with the probability $\Pi_{2 j} k_{i}$ (the anti-preferential attachment probability).

(c) Adding edge function $h^{*}(t) \cdot h^{*}(t)=h\left(p_{3}(t) r, t, k_{i}(t), \sum \Pi_{3 j} k_{i}\right)$ suggests there will be $p_{3}(t) r\left(0<p_{3}(t)<1\right)$ new edges added among a few pairs of vertices which are not linked in $N(t-1)$ at time step $t$. Two vertices linked by one new edge $j_{r}$ will be selected with the preferential attachment probability $\prod_{3 j} k_{i}$.

(d) Canceling Edges Function $z^{*}(t) \cdot z^{*}(t)=z\left(p_{4}(t) s, t, k_{i}(t), \sum \prod_{4 j} k_{i}\right)$ say that $p_{4}(t) s$ $\left(0<p_{4}(t)<1\right)$ old edges will be canceled at time step $t$. In the process, the two vertices linked by a canceled old edge $j_{s}$ will be selected with the anti-preferential attachment probability $\Pi_{4 j} k_{i}$.

(e) The external disturbance function $\varphi(t) . \varphi(t)$ gives the process of networks evolution will endure unavoidable influence from the outside world.

Suppose the dynamic evolution of a connected original network $N(0)$ having $m_{0}$ vertices is connected and then can set up a corresponding partial differential equation to explain this dynamical system, according to the independence of events. This partial 
differential equation for a degree $k_{i}(t)$ vertex in the network $N(t)$ at time step $t$ is shown as

$$
\frac{\partial k_{i}(t)}{\partial t}=f^{*}(t)+g^{*}(t)+h^{*}(t)+z^{*}(t)+\varphi(t)
$$

We are permitted to have a solution of the Eq. 3

$$
k_{i}(t)=\theta\left(t, t_{i}, \alpha_{1}, \alpha_{2}, \ldots, \alpha_{r}\right)
$$

The parameters $\alpha_{i}$ with $i=1,2, \ldots, r$ in Eq. 4 is irrelative with $t$ and $t_{i}$. Next

$$
P\left(k_{i}(t)<k\right)=P\left(t_{i}>\theta^{-1}\left(k_{i}(t), t, \beta_{1}, \beta_{2}, \ldots, \beta_{r}\right)\right)
$$

As the same result. Parameters $\alpha_{i}$ with $i=1,2, \ldots, r$ in Eq. 5 is also irrelative with $t$ and only $t_{i}$. Finally, we acquire the probability of vertices of degree $k$ in $N(t)$

$$
P(k)=\frac{\partial P\left(k_{i}(t)<k\right)}{\partial k}=\frac{\partial}{\partial k} P\left(t_{i}>\theta^{-1}\left(k_{i}(t), t, \beta_{1}, \beta_{2}, \ldots, \beta_{r}\right)\right)
$$

Based on this dynamical partial differential equation above, we will generate one tuned exponents scale-free network model in section 3. Subsection text here.

\section{The Scale-free Models}

The scale-free property of complex systems and networks have been taken much attentions from various research area $[28,29,30]$, such as physic, computer science, mathematics, chemistry, biology and economics. For uncovering the inherent principle, a mass of network models are proposed. The similarity of these models is concerned the probability.

\section{BA-model and Classic Probability}

(1) Growth: The initial model $B A_{0}$ has $m_{0}$ vertices and $n_{0}$ edges. At every time step $t$, we will add a new vertex with $m\left(m<m_{0}\right)$ edges that connect the new vertex to $m$ different ones already present in the network model. In the growth process, the number of vertices will increase continuously.

(2) Preferential attachment: The probability of a new vertex link with an old vertex $i$ in network, only depends on its degree $k_{i}$ and obeys the rule $\Pi\left(k_{i}\right)=k_{i} / \sum_{j} k_{j}$ By the continuum theory, the dynamical property of $B A$-model can be adopted and the probability that an arbitrary vertex has $k$ edges follows a power-law $P(k)=k^{-\gamma}, \gamma=3$. Fig. 1 shows an example of $B A$-model which uses the classic probability.

Based on BA-model, a mass of scale-free models are proposed to describe dynamical properties of real-life networks. They not only adopt two mechanism (growth and preferential attachment), but also enrich diversities of models by tuning the preferential attachment probability, such that

$$
\Pi\left(k_{i}\right)=\frac{k_{i}^{\omega}}{\sum_{j} k_{j}^{\omega}}, . \Pi\left(k_{i}\right)=\frac{\eta_{i} k_{i}}{\sum_{j} \eta_{j} k_{j}}, \Pi\left(k_{i}\right)=\frac{(1-p) k_{i}+p}{\sum_{j}\left((1-p) k_{j}+p\right)} .
$$

and so on. It is not hard for us that these preferential attachment probability are built on the basis of the classic probability satisfying the following three rules

(1) The number of random research objects is finite, such as the order $n_{v}(t)$ of network (the number of vertices in network) and the degree $k_{i}$ of vertex $i$. 
(2) The probability of each sample object is equal, such as the function of every edge linking each vertex is the same.

(3) If it is true that there are $k$ objects in event $A$, the probability of incident $A$ displays $P(A)=k / n$, where $n$ is the number of total objects. Obviously, it is an ideal not real to apply classic probability for exploring secret in networks.

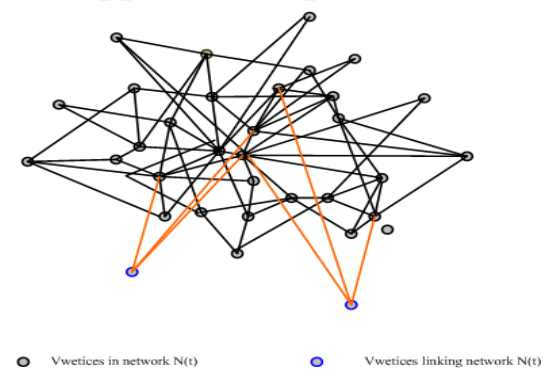

Figure-1: the $B A$-model

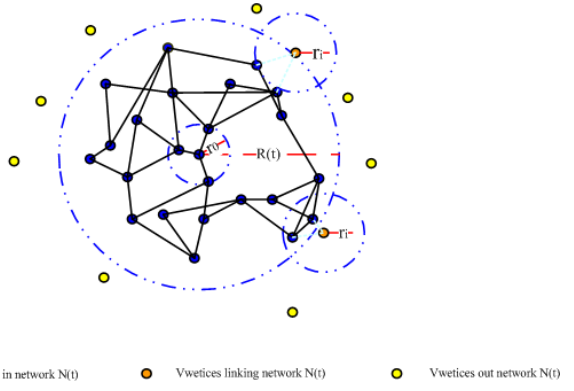

Figure-2: the $H$-model

\section{$H$-model and Geometric Probability}

In order to obtain the more proximate phenomenon being in scale-free network, a new model of WSNs, called $H$-model hereafter, has been structured by Liu et.al. [31]. The $H$-model which is similar with $B A$-model can be obtained through the following two steps.

(1) Initially $(t=0)$, the model $H_{0}$ is made up $m_{0}$ vertices and $n_{0}$ edges. At next every time step, there will be a new vertex added into networks and linked with $m$ old ones already existed in the network.

(2) When a new vertex comes into the network, it will connect to some old vertices in its local-area. The probability $\prod_{\text {local }}\left(k_{i}\right)$ of vertex $i$ can acquired a new edge from the new vertex depends on both its degree $k_{i}$ and local-area of the new (namely its radius $\left.r_{\text {new }}\right)$, which is defined as $\Pi_{\text {local }}\left(k_{i}\right)=\frac{(1-p) k_{i}+p}{\sum_{j \text { elocal }}\left((1-p) k_{j}+p\right)}, 0<p<1$. where vertex $i$ and $j$ are in the local-area of the new. By the mean-field theory, the topological degree distribution of $\mathrm{H}$-model is always playing the power-law with exponent $\gamma=3+\frac{p}{m(1-p)}$ [31]. We can get a varieties of scale-free models by adjusting the values of $m$ and $p$. Fig. 2 shows an example of $H$-model which employs the geometric probability.

The preferential attachment probability be depicted by geometric probability with some prerequisites, as follow: (1) If a random sample space $\Omega$ is full of a field, let $S_{\Omega}$ short for its measurement, such as length, area and volume. (2) The probability of each sample object dropping into the same sub-area is equal. (3) If let $S_{A}$ be the measurement of $A$ which is a sub-area of $\Omega$, the probability is $P(A)=S_{A} / S_{\Omega}$.

\section{New Model and Its Degree Distribution}

Motivated from $B A$-model and $H$-model shown above, we will present a social-network model $N(t)$ whose dynamical evolution includes both the classic and geometric probabilities, where vertices and edges are standed for people and their relationships, respectively.

(a) For $t=0$, staring with $m_{0}$ vertices and $n_{0}$ edges, at every time step, $a$ new vertices with $m$ edges take part in the social-network $N(t)$. 
(b) A new vertices is made from three kinds different ones. The first ones stand for some social-talent who have more chance to making friends with celebrities. In network models, by using the classic probability, they always link to those old vertices having more lager degree as soon as possible. The second is an ordinary man who can meet with some people in limited social-circle. Most of people living in society belong to this part. Similarity, we can portray this way of communicating by the geometric probability. Others can be named wanders who are free to associate with another people. It is clear that the number of aimless people is yet more smaller. This probability is random in our theoretical analysis.

These replication and connection steps can be repeated indefinitely. We will use the following notations [20]: $V(t)$ and $E(t)$ denote, the sets of vertices and edges of the $N(t)$, respectively. As follow, we say $v_{t}=|V(t)|$ and $e_{t}=|E(t)|$ to be the numbers of vertices and edges of $N(t)$. After time step $t$, we can know $v_{t}=a t+n_{0}$ and $e_{t}=a m t+m_{0}$. Suppose that the numbers of part one, part two and another are $\alpha_{1} a, \alpha_{2} a$ and $\alpha_{3} a$, separately. Assume that $k_{i}$ is a continuous real variable. According to its dynamical evolution mechanism and the mean-field theory

$$
\Pi\left(k_{i}\right)=\prod_{\text {whole }}\left(k_{i}\right)+\prod_{\text {local }}\left(k_{i}\right)+\prod_{\text {random }}\left(k_{i}\right)=\frac{\left(1-p_{1}\right) k_{i}+p_{1}}{\sum_{j}\left(1-p_{1}\right) k_{j}+p_{1}}+\frac{\left(1-p_{2}\right) k_{i}+p_{2}}{\sum_{j \text { elocal }}\left(1-p_{2}\right) k_{j}+p_{2}}+\frac{1}{v_{t}}
$$

Consequently, $k_{i}$ satisfies the dynamical equation

$$
\frac{\partial k_{i}(t)}{\partial t}=\frac{\alpha_{1} a m\left[\left(1-p_{1}\right) k_{i}+p_{1}\right]}{\sum_{j}\left(1-p_{1}\right) k_{j}+p_{1}}+\frac{\left.\alpha_{2} a m\left[1-p_{2}\right) k_{i}+p_{2}\right]}{\sum_{j \in l o c a l}\left(1-p_{2}\right) k_{j}+p_{2}}+\frac{\alpha_{3} a m}{v_{t}}
$$

For the purpose of statement, if we set $A_{1}=\alpha_{1} a m\left(1-p_{1}\right), A_{2}=2\left(1-p_{1}\right) a m+a m p_{1}$, $A_{3}=\alpha_{1} a m p_{1}, A_{4}=2\left(1-p_{1}\right)\left(m_{0}-a m\right)+p_{1}\left(n_{0}-a\right), \quad B_{1}=\alpha_{2} a m\left(1-p_{2}\right), \quad B_{2}=2\left(1-p_{2}\right) a m+a m p_{2}$, $B_{3}=\alpha_{2} a m p_{2}, B_{4}=2\left(1-p_{2}\right)\left(m_{0}-a m\right)+p_{2}\left(n_{0}-a\right)$. As $t \rightarrow \infty$, the Eq. 7 is

$$
\frac{\partial k_{i}(t)}{\partial t}=\frac{A_{1} k_{i}+A_{3}}{A_{2} t+A_{4}}+\frac{B_{1} k_{i}+B_{3}}{B_{2} t+B_{4}}+\frac{\alpha_{3} a m}{a(t-1)+n_{0}} \approx \frac{A_{1} k_{i}+A_{3}}{A_{2} t}+\frac{B_{1} k_{i}+B_{3}}{B_{2} t}+\frac{\alpha_{3} a m}{a t}=\frac{\left(A_{2} B_{1}+B_{2} A_{1}\right) k_{i}+C}{A_{2} B_{2} t}
$$

where $C=B_{3} A_{2}+B_{2} A_{3}+A_{2} B_{2} \alpha_{3} m$. The solution of the Eq. 8,with the initial condition that each vertex $i$ at its introduction has $k_{i}\left(t_{i}\right)=m$, is

$$
k_{i}(t)=\left(m+\frac{C}{B_{1} A_{2}+B_{2} A_{1}}\right)\left(\frac{t}{t_{i}}\right)^{-\beta}-\frac{C}{B_{1} A_{2}+B_{2} A_{1}}, \quad \beta=\frac{A_{2} B_{1}+B_{2} A_{1}}{A_{2} B_{2}}
$$

By Eq. 9, the probability $P\left(k_{i}(t)<k\right)$ that one vertex has a degree $k_{i}(t)$ smaller than $k$ can be written as

$$
P\left(k_{i}(t)<k\right)=P\left(t_{i}>t\left[\frac{\left(A_{2} B_{1}+B_{2} A_{1}\right) k+C}{\left(A_{2} B_{1}+B_{2} A_{1}\right) m+C}\right]^{-1 / \beta}\right)
$$

Suppose that we add these new vertices at equal time interval into the network by $P\left(t_{i}\right)=\left(m_{0}+t_{i}\right)^{-1}$, The degree distribution $P(k)$ can be obtained as follows

$$
P(k)=\frac{\partial P\left(k_{i}(t)<k\right)}{\partial k}=\frac{t}{m_{0}+t} \frac{1}{\beta}\left[\frac{\left(A_{2} B_{1}+B_{2} A_{1}\right) k+C}{\left(A_{2} B_{1}+B_{2} A_{1}\right) m+C}\right]^{-(1 / \beta+1)}
$$

According to Eq. 11, the topological degree distribution of the social-network model $N(t)$ obeys the power-law formula (1) with exponent $\gamma=1+1 / \beta$ The value range of the 
power law exponent $\gamma$ will be various. As shown in Fig. 3, Fig. 4 and Fig. 5. We will calculate the Eq. 7 by the cumulation

$$
\sum\left|V\left(k_{i}, t\right)\right| \frac{\partial k_{i}(t)}{\partial t}
$$

The mark $\left|V\left(k_{i}, t\right)\right|$ stands the number of vertices having degree $k_{i}$ at time step $t$. The reason why this Eq. 12 attends to a constant value is closely related to the regularity of probability. Based on these analysis above, $N(t)$ is a scale-free network model.

\section{Conclusion}

According to a mass of research results on networks at present, we firstly set up the general partial differential equation with 5 characteristic functions and achieve its solutions by abstract numerical analysis under a special initial condition. Secondly, we consider the relationship of both the classic probability and BA-model and the connection between the geometric probability and $H$-model. Motivated from $B A$-model and $H$-model, we establish one social-network model which involves the classic and geometric probability. By the continuum theory, it follows the power-law and has scale-free feature. Although we have gotten the exact solution about the degree distribution of it, we only think about the entering vertices function $f^{*}(t)$ in Eq. 3, hence no excellent results for the real dynamic evolution. In addition, future work on these and other problems would help to highlight the unusual behavior of these models in the future $[19,32,33,34,35,36]$.

Problem: Bayesian probability model: In a growing network model, it will be named as Bayesian model if the preferential attachment probability is Bayesian's one. This discussion about network model and Bayesian probability may attract people's attentions.

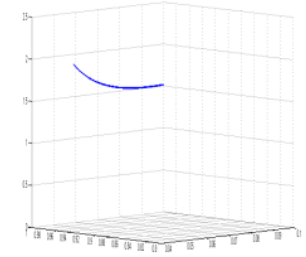

Figure-3: The relation between the degree distribution exponent $\gamma$ of the model $N(t)$ and parameters $\alpha_{1}$ and $\alpha_{2}$.

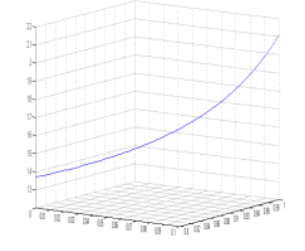

Figure-4: The relation between the degree distribution exponent $\gamma$ of the model $N(t)$ and two probabilities $p_{1}$ and $p_{2}$.

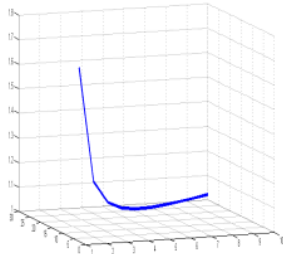

Figure-5: The relation between the degree distribution exponent $\gamma$ of the model $N(t)$ and $a$ the number of new vertices added and $m$ being the number of edges linking each new vertex added .

\section{Acknowledgement}

This research was supported by the National Natural Science Foundation of China under grants No. 61163054, No. 61363060 and No. 61662066.

\section{References}

[1] B. A. Huberman, The laws of the Web (MIT Press, Cambridgem MA, USA, 2001)

[2] M. E. Newman, Phys. Rev. E 64, 016131 (2001) 
[3] D. Garlaschelli, G. Caldarelli, L. Pietronero, Nature 423, 165(2003)

[4] J. M. Montoya, R. V. Sole, J. Theor. Biol. 214, 405 (2002)

[5] A. Wagner, Mol. Biol. Evol. 18, 1283 (2001)

[6] H. Jong, B. Tombor, R. Albert, Z. N. Oltvai, A. -L. Barabási. Nature 407, 651 (2000)

[7] S. N. Dorogovtsev, A. V. Goltsev, J. F. F. Mendes. Pseudofractal scale-free web. Physical reviewer, 2002, (65), 066122-066125

[8] S. N. Dorogovtsev, A. V. Goltsev, J. F. F. Mendes. Pseudofractal scale-free web. Physical reviewer, 2002, (65), 066122-066125

[9] A. -L. Barabási, E. Ravasz, and T. Vicsek. Physica A 299, 559 (2001)

[10] E. Agliari, R. Burioni.Phys. Rev. E 80, 031125(2009)

[11] T. Nishikawa, A. E. Motter, Y. -C. Lai, F. C. Hoppensteadt. Phys. Lett. 91, 014101(2003)

[12] S. Jung, S. Kim, B. Kahng, Phys. Rev. E 65, 056101(2002)

[13] F. Chung, L. Lu. Proc. Natl. Acad. Sic. U.S.A 99, 15879 (2002)

[14] R. Cohen, S. Havlin. Phys. Rev. Leet. 90. 058701 (2003)

[15] Erdös P, Rényi A. On random graphs. Publ. Math. , 1959, 6: 290-297

[16] D. J. Watts and S. H. Strogatz, Nature 393 (1998)

[17] A. -L. Barabási, R. Albert, H. Jeong Mean-field theory for scale-free random networks Physica A 272, 173-187 (1999)

[18] D. J. Watts, Small Worlds: The Dynamics of Networks between Order and Randomness (Princeton University Press, Princeton, NJ, 1999)

[19] B. Yao, M. Yao, X, E, Chen, X. Liu, W. J. Zhang, J, Applied Mechanics and Materials, Volumes 513-517, 2014

[20] J. Adrian Bondy and U. S. R. Murty, Graph Theory with Application, M. The MaCmillan Press Itd, London and Basingstoke, New York, 1976

[21] C. M. Song, T. Koren, P. Wang, A. L. Barabási, Modelling the scaling properties of human mobility, J. Nature Physics. 1760 (2010) 1-6

[22] F.Q Gu, S.H Fan.The two-way evolution model of a BA scale-free network,J.

Journal of Jinan University. 2013(5)

[23] L.H Ma, L.G Zhang, J.K Li, S.C Yang.The degree distribution of scale-free and collaboration network model,J. Journal of Hebei University of technology. 2011(4)

[24] Chen Qinghua, Shi Dinghua. Physica A,2004, 335:240-248

[25] H.Z Liang, H.X Yao, X.B Zhang.Characters analysis for a class of scale-free networks,J. Complex system and complexity science. 2005(3)

[26] F. Ma, J. Su, B. Yao, M. Yao, Mechanical and Electronic Engineering Conference (JIMEC 2016), ISSN 2352-5401, Volume 59, pp155-162. 
[27] B. Yao, F. Ma, J. Su, X. M. Wang, X. Y. Zhao, M. Yao, Proceedings of 2016 IEEE Advanced Information Management, Communicates,Electronic and Automation Control Conference (IMCEC 2016) pp549-554.

[28] X. L Jia, S. H Cai, F. R Zhang.A dynamic scale-free network model,J. Journal of Sichuan Normal University. 2009(6)

[29] G. Yan, G. Tsekenis, B. Barzel, J. J Slotine, Y. Y. Liu, A. L. Barabási, J. Nature Physics. 3422 (2015) 779-786

[30] Charo. I. Del. Genio, Thilo. Gross, Kevin. E. Bassler, J. Physical review letters. 178701 (2011) 1-4

[31] Haoran. Liu, Rongrong. Yin, Bin. Liu, Yaqian. Li, J. Computers and Electrical Engineering. 000 (2016) 1-11

[32] B. Yao, X. Liu, W. J. Zhang, X. E. Chen, X. M. Zhang, M.Yao, Z. X. Zhang, 2013 IEEE International Conference on High Perfermance Computing and Communication, 2354-2361

[33] B. Yao, M. Yao, X. E. Chen, X. Liu, W. J. Zhang. Applied Meachanics and Materials,Vol. 513-517, 2013, 2444-2448

[34] B.Yao, C. Yang, M. Yao, H. Y. Wang, X. E. Chen, X. M. Zhang, M. G. Li. Applied Mechanics and Materials Vol.380-384(2013),2034-2037.

[35] B. Yao, X. M. Wang, J. Su, F. Ma, X. Y. Zhao, M. Yao, M. J. Zhang, G. X. Wang, Joint International Information Technology, Mechanical and Electronic Engineering Conference (JIMEC 2016), ISSN 2352-5401, Volume 59, pp57-61.

[36] J. Su, F. Ma, B. Yao, M. Yao, Joint International Information Technology, Mechanical and Electronic Engineering Conference (JIMEC 2016), ISSN 2352-5401, Volume 59, pp260-265. 